\title{
POLARIZED 4-MANIFOLDS, EXTREMAL KÄHLER METRICS, AND SEIBERG-WITTEN THEORY
}

\author{
Claude LeBrun
}

\begin{abstract}
Using Seiberg-Witten theory, it is shown that any Kähler metric of constant negative scalar curvature on a compact 4-manifold $M$ minimizes the $L^{2}$-norm of scalar curvature among Riemannian metrics compatible with a fixed decomposition $H^{2}(M)=H^{+} \oplus H^{-}$. This implies, for example, that any such metric on a minimal ruled surface must be locally symmetric.
\end{abstract}

\section{Introduction}

In the late 1950's, Calabi first posed the problem of representing each Kähler class on a compact complex manifold by a Kähler metric of constant scalar curvature. This eventually led him [2] to define extremal Kähler metrics, which minimize the functional $\int s^{2} d \mu$ over a fixed Kähler class; here $s$ denotes the scalar curvature, and $d \mu$ denotes the metric volume measure. Any Kähler metric of constant scalar curvature is extremal in this sense, but Calabi showed by example that the converse is generally false.

In real dimension 4, new insights into this problem can be gained by temporarily venturing outside the Kählerian arena, and instead working in a broader Riemannian context. Instead of fixing a Kähler class, we will fix a closely related direct sum decomposition $H^{2}(M, \mathbb{R})=H^{+} \oplus H^{-}$. Seiberg-Witten theory will then allow us to see that any Kähler metric of constant negative scalar curvature is an absolute minimum of $\int s^{2} d \mu$ among metrics compatible with such a decomposition. As an application, we will then classify Kähler metrics of constant negative scalar curvature on minimal ruled surfaces.

\section{Polarizations}

Definition 1. Let $M$ be a smooth compact oriented 4-manifold. A polarization of $M$ is a maximal linear subspace $H^{+} \subset H^{2}(M, \mathbb{R})$ for which the restriction of the intersection form is positive definite.

Received June 8, 1995.

Supported in part by NSF grant DMS-9505744. 
Because the intersection form is non-degenerate, every polarization determines an orthogonal complement $H^{-}$with respect to the intersection form, and the intersection form is negative definite on this orthogonal complement; this puts polarizations of $M$ and of the reverse-oriented manifold $\bar{M}$ in natural one-to-one correspondence. The dimensions $b_{ \pm}:=\operatorname{dim} H^{ \pm}$ are important homeomorphism invariants of $M$, and their difference $\tau=$ $b_{+}-b_{-}$is called the signature. Given a polarization, we will routinely invoke the decomposition

$$
H^{2}(M)=H^{+} \oplus H^{-}
$$

to uniquely express elements $\alpha \in H^{2}$ as $\alpha=\alpha^{+}+\alpha^{-}$, where $\alpha^{ \pm} \in H^{ \pm}$.

While the imposition of a polarization may seem frivolous, such choices arise quite naturally in Riemannian geometry. Indeed, if $g$ is a smooth Riemannian metric on $M$, then the space

$$
H^{+}(g):=\left\{[\varphi] \in H^{2}(M) \mid \varphi \in C^{\infty}\left(\wedge^{2}\right)\right\}
$$

of self-dual $g$-harmonic 2 -forms is a polarization on $M$. We will say that a Riemannian metric $g$ is adapted to the polarization $H^{+}$if $H^{+}(g)=H^{+}$. A polarization will be called a metric polarization if there is at least one metric adapted to it.

Example. Let $(M, J, g)$ be a compact Kähler manifold of complex dimension 2, and let $\omega$ denote the associated Kähler form. Let $\Re e H^{2,0} \subset$ $H^{2}(M, \mathbb{R})$ denote the de Rham classes which are represented by real parts of holomorphic 2-forms. Then $H^{+}(g)=\mathbb{R}[\omega] \oplus \Re e H^{2,0}$.

Algebraic geometers sometimes use the term "polarization" to denote a choice of Kähler class $[\omega]$ on a compact complex manifold $(M, J)$. In light of the above example, our terminology may thus be justified by the fact that the polarization $H^{+}(g)$ of a Kähler metric determines the Kähler class $[\omega]$ if the complex structure $J$ and total volume $[\omega]^{2} / 2$ are already known.

Because the Hodge star operator is conformally invariant on middle dimensional forms, the present notion of polarization is conformally invariant; that is, $H^{+}(g)=H^{+}(f g)$ for any smooth positive function $f$. Thus all our conclusions about metrics adapted to a fixed polarization will also imply results about global conformal invariants.

\section{Seiberg-Witten Theory}

Let $M$ be a smooth connected compact oriented 4-manifold, and assume that $M$ admits an orientation-compatible almost-complex structure $J: T M \rightarrow T M, J^{2}=-1$. Such an almost-complex structure determines a $\operatorname{spin}^{c}$ structure on $M$, meaning a cohomology class $c \in H^{2}(F, \mathbb{Z})$ on the 
oriented frame bundle $F \rightarrow M$ whose restriction of $c$ to a typical fiber $F_{x} \cong S L(4, \mathbb{R}) \times \mathbb{R}$ is the nonzero element of $H^{2}\left(F_{x}, \mathbb{Z}\right) \cong \mathbb{Z}_{2}$. Indeed, if $g$ is any $J$-invariant Riemannian metric on $M$, let $F_{S O} \subset F$ be the bundle of oriented $g$-orthogonal frames, and let $F_{U} \subset F_{S O}$ denote the bundle of unitary frames with respect to $g$ and $J$. Then the Poincaré dual of the submanifold $F_{U} \subset F_{S O}$, thought of as an element of $H^{2}(F, \mathbb{Z})=H^{2}\left(F_{S O}, \mathbb{Z}\right)$, is fiberwise nonzero and is independent of $g$; this is the promised $\operatorname{spin}^{c}$ structure $c$. If a $\operatorname{spin}^{c}$ structure arises in this way, we will say that it is of almost-complex type, and we will say that the almost-complex structure $J$ and the $\operatorname{spin}^{c}$ structure $c$ are compatible. One may choose to think of a $\operatorname{spin}^{c}$ structure of almost-complex type as an equivalence class of almost-complex structures $J$; two such structures are then equivalent iff their graphs are homologous as submanifolds of the bundle $F / G L(2, \mathbb{C})$ of orientation-compatible almost-complex structures.

Any $\operatorname{spin}^{c}$ structure on $M$ determines, up to isomorphism, a complex line bundle $L \rightarrow M$ such that $c_{1}(L) \equiv w_{2}(T M) \bmod 2$, by setting $c_{1}(L)=2 c \in H^{2}(M, \mathbb{Z}) \subset H^{2}(F, \mathbb{Z})$; conversely any such choice of $c_{1}(L) \in H^{2}(M, \mathbb{Z})$ determines a $\operatorname{spin}^{c}$ structure up to 2-torsion in $H^{2}(M, \mathbb{Z})$. If we choose a Riemannian metric $g$ on $M$, a $\operatorname{spin}^{c}$ structure determines rank 2 Hermitian vector bundles $V_{ \pm} \rightarrow M$ with $\wedge^{2} V_{ \pm}=L$ and $T^{*} M \otimes \mathbb{C} \cong \operatorname{Hom}\left(V_{+}, V_{-}\right)$; and on any contractible open set in $M$ we have canonical (sign-ambiguous) isomorphisms

$$
V_{ \pm}=\mathbb{S}_{ \pm} \otimes L^{1 / 2}
$$

where $\mathbb{S}_{ \pm}$are the left- and right-handed spinor bundles of $g$, and $L^{1 / 2}$ is a complex line bundle whose square is $L$. Each unitary connection $A$ on $L$ therefore induces a unitary connection $\nabla_{A}: C^{\infty}\left(V_{+}\right) \rightarrow C^{\infty}\left(T^{*} M \oplus V_{+}\right)$ on $V_{+}$, and following this with the isomorphism $T^{*} M \otimes \mathbb{C} \cong \operatorname{Hom}\left(V_{+}, V_{-}\right)$ gives us a Dirac operator $D_{A}: C^{\infty}\left(V_{+}\right) \rightarrow C^{\infty}\left(V_{-}\right)$.

This can all be made much more concrete for $\operatorname{spin}^{c}$ structures of almostcomplex type. Given a Riemannian metric $g$ on $M$, we can represent such a $\operatorname{spin}^{c}$ structure by an almost-complex structure $J: T M \rightarrow T M$, $J^{2}=-1$ such that $J^{*} g=g$. The tangent bundle $T M$ of $M$ is thereby given the structure of a rank 2 complex vector bundle $T^{1,0}$ by defining scalar multiplication by $i$ to be $J$. Setting $\wedge^{0, p}:=\wedge^{p}{\overline{T^{1,0}}}^{*} \cong \wedge^{p} T^{1,0}$, the bundles $V_{ \pm}$of twisted spinors are given by

$$
\begin{aligned}
& V_{+}=\wedge^{0,0} \oplus \wedge^{0,2} \\
& V_{-}=\wedge^{0,1}
\end{aligned}
$$

and their Hermitian structures are the obvious ones induced by $g$. In 
particular, $L$ is the anti-canonical bundle of $(M, J)$, and we therefore have

$$
c_{1}(L)^{2}=(2 \chi+3 \tau)(M) .
$$

Spin $^{c}$ structures of almost-complex type are characterized by this last property.

If $(M, g, J)$ is a Kähler manifold, so that $J$ is parallel with respect to the metric connection, and if $A$ is the so-called Chern connection on the anticanonical bundle $L$, then the connection $\nabla_{A}$ on $V_{+}$has parallel sections corresponding to the constant sections of $\wedge^{0,0} \subset V_{+}$. Conversely, a metric is Kähler for some $c$-compatible complex structure $J$ if there is a choice of $A$ for which $V_{+}$has a nontrivial parallel section, since this implies that the holonomy of $\mathbb{S}_{+}$is contained in $U(1) \subset S U(2)$, the Riemannian holonomy is then contained in $(U(1) \times S U(2)) / \mathbb{Z}_{2}=U(2)$. For $g$ a Kähler metric and $A$ the Chern connection, the Dirac operator can correspondingly be expressed as $D_{A}=\sqrt{2}\left(\bar{\partial} \oplus \bar{\partial}^{*}\right)$, where $\bar{\partial}: C^{\infty}\left(\wedge^{0,0}\right) \rightarrow C^{\infty}\left(\wedge^{0,1}\right)$ is the $J$-antilinear part of the exterior differential $d$, acting on complex valued functions, and where $\bar{\partial}^{*}: C^{\infty}\left(\wedge^{0,2}\right) \rightarrow C^{\infty}\left(\wedge^{0,1}\right)$ is the formal adjoint of the map induced by the exterior differential $d$ acting on 1-forms; more generally, $D_{A}$ will differ from $\sqrt{2}\left(\bar{\partial} \oplus \bar{\partial}^{*}\right)$ by only $0^{\text {th }}$ order terms.

Let us now fix a $\operatorname{spin}^{c}$ structure $c$ of almost-complex type on on $M$. For each Riemannian metric $g$, the Seiberg-Witten equations [10]

$$
\begin{aligned}
D_{A} \Phi & =0 \\
F_{A}^{+} & =i \sigma(\Phi)
\end{aligned}
$$

are then equations for an unknown smooth connection $A$ on $L$ and an unknown smooth section $\Phi$ of $V_{+}$. Here the purely imaginary 2-form $F_{A}^{+}$ is the self-dual part of the curvature of $A$, and the natural real quadratic map $\sigma: V_{+} \rightarrow \wedge_{+}^{2}$ satisfies $|\sigma(\Phi)|^{2}=|\Phi|^{4} / 8$. For our purposes, it is crucial that equations (3) and (4) imply the Weitzenböck formula

$$
\nabla_{A}^{*} \nabla_{A} \Phi+\frac{s+|\Phi|^{2}}{4} \Phi=0
$$

Given a solution $(A, \Phi)$ of (3) and (4) and a smooth map $f: M \rightarrow S^{1} \subset \mathbb{C}$, the pair $(\hat{A}, \hat{\Phi})=\left(A-2 f^{-1} d f, f \Phi\right)$ is also a solution; solutions which are related in this way are called gauge equivalent. A solution is called reducible if $\Phi \equiv 0$; otherwise, it is called irreducible.

Now, in addition to such a $\operatorname{spin}^{c}$ structure $c$, let us fix a metric polarization $H^{+}$on $M$. Assume that $c_{1}^{+}:=\left[c_{1}(L)\right]^{+} \in H^{+}$is nonzero, which guarantees that every solution of the Seiberg-Witten equations is irreducible whenever $g$ is an $H^{+}$-adapted metric. For each such metric, one can then define the Seiberg-Witten invariant $n_{c}(M, g) \in \mathbb{Z}$ by counting the solutions 
modulo gauge equivalence with appropriate multiplicities for a generic small perturbation of (3) and (4); in particular, $n_{c}(M, g) \neq 0$ implies there is a solution of $(3)$ and (4). We now define $n_{c}\left(M, H^{+}\right)=n_{c}(M, g)$ for any $H^{+}$-adapted metric $g$. This is metric independent because the moduli spaces corresponding to different $H^{+}$-adapted metrics are cobordant as oriented 0 -manifolds. Indeed, when $b_{+}>1, n_{c}\left(M, H^{+}\right)$is even independent of the polarization. By contrast, when $b_{+}=1$, the invariant generally jumps [3, 4] as $H^{+}$passes though polarizations for which $c_{1}^{+}=0$.

The following result $[3,6,9,10]$ shows that the invariant is nontrivial for many interesting polarized manifolds:

Theorem 1. Let $(M, J, g)$ be a compact Kähler surface for which the Kähler class $[\omega]$ satisfies $c_{1} \cdot[\omega]<0$. Let $H^{+}=H^{+}(g)$ be the metric polarization, and let $c$ be the canonical spin structure of $(M, J)$. Then $n_{c}\left(M, H^{+}\right)=1$.

The assumption that $c_{1} \cdot[\omega]<0$ amounts to the requirement that the scalar curvature $s$ of $(M, g)$ be negative "on average," by virtue of the Gauss-Bonnet type formula $\int_{M} s d \mu=4 \pi c_{1} \cdot[\omega]$. In fact, the proof of Theorem 1 becomes particularly simple [5] if the scalar curvature is assumed to be a negative constant.

The following scalar-curvature inequality is the crux of the the present note:

Theorem 2. Let $\left(M, H^{+}\right)$be a smooth compact oriented 4-manifold which is polarized. Suppose that there is a spin ${ }^{c}$ structure $c$ of almost-complex type on $M$ for which the Seiberg-Witten invariant is nonzero; let $c_{1}(L) \in$ $H^{2}(M, \mathbb{R})$ denote the anti-canonical class of this structure, and let $c_{1}^{+} \neq 0$ be its orthogonal projection to $\mathrm{H}^{+}$with respect to the intersection form. Then every $\mathrm{H}^{+}$-adapted Riemannian metric $g$ satisfies

$$
\int_{M} s^{2} d \mu \geq 32 \pi^{2}\left(c_{1}^{+}\right)^{2}
$$

with equality iff $g$ is Kähler with respect to a c-compatible complex structure and has constant negative scalar curvature.

Proof. For any given metric $g$ adapted to $H^{+}$, there must exist an irreducible solution of (3) and (4), since otherwise we would have $n_{c}\left(M, H^{+}\right)=$ 0 . Now the Weitzenböck formula 5 tells us that

$$
0=\int_{M}\left(4|\nabla \Phi|^{2}+s|\Phi|^{2}+|\Phi|^{4}\right) d \mu
$$


so that $\int(-s)|\Phi|^{2} d \mu \geq \int|\Phi|^{4} d \mu$, with equality iff $\Phi$ is parallel. The Schwartz inequality therefore tells us that

$$
\int_{M} s^{2} d \mu \geq \frac{\left(\int_{M}(-s)|\Phi|^{2} d \mu\right)^{2}}{\int_{M}|\Phi|^{4} d \mu} \geq \int_{M}|\Phi|^{4} d \mu
$$

with equality iff $\nabla \Phi=0$ and $s$ is constant. On the other hand, $\left|F_{A}^{+}\right|^{2}=$ $|\sigma(\Phi)|^{2}=|\Phi|^{4} / 8$, so this may be rewritten as

$$
\int_{M} s^{2} d \mu \geq 8 \int_{M}\left|F_{A}^{+}\right|^{2} d \mu .
$$

But now letting $\varphi$ denote the harmonic representative of the de Rham class $\left[F_{A}\right]=2 \pi c_{1}$, we have

$$
\begin{aligned}
\int_{M}\left|F_{A}^{+}\right|^{2} d \mu & =\frac{1}{2} \int_{M}\left(\left|F_{A}^{+}\right|^{2}-\left|F_{A}^{-}\right|^{2}\right) d \mu+\frac{1}{2} \int_{M}\left(\left|F_{A}^{+}\right|^{2}+\left|F_{A}^{-}\right|^{2}\right) d \mu \\
& =2 \pi^{2} c_{1}(L)^{2}+\frac{1}{2} \int_{M}\left|F_{A}\right|^{2} d \mu \\
& \geq 2 \pi^{2} c_{1}(L)^{2}+\frac{1}{2} \int_{M}|\varphi|^{2} d \mu \\
& =\frac{1}{2} \int_{M}\left(\left|\varphi^{+}\right|^{2}-\left|\varphi^{-}\right|^{2}\right) d \mu+\frac{1}{2} \int_{M}\left(\left|\varphi^{+}\right|^{2}+\left|\varphi^{-}\right|^{2}\right) d \mu \\
& =\int_{M}\left|\varphi^{+}\right|^{2} d \mu \\
& =4 \pi^{2}\left(c_{1}^{+}\right)^{2}
\end{aligned}
$$

because a harmonic form minimizes the $L^{2}$ norm among closed forms in its deRham class. Hence

$$
\int_{M} s^{2} d \mu \geq 32 \pi^{2}\left(c_{1}^{+}\right)^{2}
$$

as claimed. Moreover, equality is achieved only if $s$ is constant and $\nabla \Phi=0$, which implies that $g$ is Kähler with respect to a $c$-compatible complex structure.

Conversely, any Kähler metric with constant scalar curvature will saturate this bound, since $\varphi^{+}=s \omega / 4$ and $d \mu=\left|\omega^{2} / 2\right|$ for such a metric.

Notice that the above inequality will hold, more generally, for any metric and $\operatorname{spin}^{c}$ structure for which there is an irreducible solution of the SeibergWitten equations. Thus, while it is also possible in principal to define Seiberg-Witten invariants for $\operatorname{spin}^{c}$ structures which are not of almostcomplex type, these can often be shown to vanish by producing metrics for which $\int s^{2} d \mu$ is sufficiently small. 
The above result has a curious ramification for conformal geometry. Let $g$ be a Kähler metric of constant negative scalar curvature on a compact complex surface $M$. Let $[g]$ be the conformal class of $g$, and let $[g]_{-} \subset[g]$ be the open subset of metrics of negative scalar curvature. Then $g$ simultaneously minimizes $\int s^{2} d \mu$ and maximizes $\left(\int s d \mu\right)^{2} / \int d \mu$, considered as functionals on $[g]_{-}$!

\section{Ruled Surfaces}

While the best-understood obstructions to the existence of Kähler metrics of constant scalar curvature entail the existence of nontrivial holomorphic vector fields, a more subtle obstruction, related to Mumford stability, was discovered by Burns and de Bartolomeis [1]. While their result deals only with $s \equiv 0$ metrics on minimal ruled surfaces, its formulation is so elegant as to make it desirable to put this isolated result in a wider context. We shall now take a small step in this direction by showing that analogous conclusions hold for Kähler metrics of constant negative scalar curvature on minimal ruled surfaces.

Theorem 3. Let $M$ be the total space of an oriented 2-sphere bundle $M \rightarrow$ $\Sigma$ over a compact oriented surface. For some complex structure $J$, suppose that there is a Kähler metric $g$ of constant negative scalar curvature on $M$. Then the universal cover of $(M, g)$ is isometric to the product $S^{2} \times \mathcal{H}^{2}$, where the 2-sphere and hyperbolic 2-space are endowed with appropriate constant multiples of their standard metrics.

Proof. First observe that the structure group $\operatorname{Diff}^{+}\left(S^{2}\right)$ of $M \rightarrow \Sigma$ can be reduced to $S O(3)$, since the induced metric on each fiber $S^{2}$ can be conformally rescaled to yield a metric of curvature +1 , and the freedom in doing so is paramaterized by the contractible space $S L(2, \mathbb{C}) / S U(2)$. As a consequence, $M$ admits a fiberwise antipodal map $\psi: M \rightarrow M$. Since this is orientation reversing, we may define a $\operatorname{spin}^{c}$ structure $\bar{c}$ on the reverseoriented manifold $\bar{M}$ by setting $\bar{c}=\psi^{*} c$, where $c$ is the $\operatorname{spin}^{c}$ structure on $M$ induced by $J$.

Because $\psi$ reverses orientation, $\psi^{*}: H^{2}(M) \rightarrow H^{2}(M)$ reverses the sign of the intersection form:

$$
\psi^{*}(\alpha) \cdot \psi^{*}(\beta)=-\alpha \cdot \beta \forall \alpha, \beta \in H^{2}(M, \mathbb{R}) .
$$

Since $\left(\psi^{*}\right)^{2}=\left(\psi^{2}\right)^{*}=1$, it follows that $\alpha \cdot \psi^{*}(\alpha)=0$. But $H^{2}(M, \mathbb{R})$ is 2-dimensional, and $\psi^{*}$ therefore takes any 1-dimensional subspace to its intersection-form orthogonal subspace. This shows that $\psi^{*}\left(H^{+}(g)\right)=$ $H^{-}(g)$. 
Since we therefore know that $\left(M, H^{+}(g), c\right)$ and $\left(\bar{M}, H^{-}(g), \bar{c}\right)$ are isomorphic as polarized oriented 4-manifolds with $\operatorname{spin}^{c}$ structure, they have the same Seiberg-Witten invariant, and

$$
\left(c_{1}^{+}\right)^{2}\left(M, H^{+}(g), c\right)=\left(c_{1}^{+}\right)^{2}\left(\bar{M}, H^{-}(g), \bar{c}\right) .
$$

But $g$ is a Kähler metric with constant negative scalar curvature, implying that $n_{c}\left(M, H^{+}(g)\right)=1$ and $\int_{M} s^{2} d \mu=32 \pi^{2}\left(c_{1}^{+}\right)^{2}\left(M, H^{+}(g), c\right)$. Hence $n_{c}\left(\bar{M}, H^{-}(g)\right)=1$ and $\int_{M} s^{2} d \mu=32 \pi^{2}\left(c_{1}^{+}\right)^{2}\left(\bar{M}, H^{-}(g), \bar{c}\right)$, too. By Theorem 2, the latter implies that $g$ is Kähler with respect to a complex structure $\tilde{J}$ compatible with $\bar{c}$, and hence compatible with the orientation of $\bar{M}$.

Now the $g$-preserving linear maps $J$ and $\tilde{J}$ lie in opposite factors of $S O(4)=(S U(2) \times S U(2)) / \mathbb{Z}_{2}$, and so commute. The endomorphism $J \tilde{J}=\tilde{J} J$ is therefore diagonalizable, with eigenvalues \pm 1 , and we have an eigenspace decomposition $T M=L_{1} \oplus L_{2}$ of the tangent bundle into rank 2 real vector bundles. Since both complex structures are invariant under parallel transport with respect to $g$, this decomposition is parallel, and the universal cover of $(M, g)$ is therefore the Riemannian product $\left(X_{1}, g_{1}\right) \times\left(X_{2}, g_{2}\right)$ of a pair of complete simply connected surfaces. Since the scalar curvature $s$ of $g$ is constant, and since $s$ is the sum $s_{1}+s_{2}$ of the scalar curvatures of $\left(X_{1}, g_{1}\right)$ and $\left(X_{2}, g_{2}\right)$, it follows that $s_{1}$ and $s_{2}$ must both be constant. Now $\pi_{3}(\Sigma)$ is finite, so the exact homotopy sequence

$$
\cdots \rightarrow \pi_{3}(\Sigma) \rightarrow \pi_{2}\left(S^{2}\right) \rightarrow \pi_{2}(M) \rightarrow \pi_{2}(\Sigma) \rightarrow \cdots
$$

predicts that $\pi_{2}(M) \neq 0$; passing to the universal cover, we thus have $\pi_{2}\left(X_{1}\right) \times \pi_{2}\left(X_{2}\right)=\pi_{2}\left(X_{1} \times X_{2}\right)=\pi_{2}(M) \neq 0$, and at least one of the simply connected surfaces $X_{j}$ must therefore be a 2 -sphere. On the other hand, $s=s_{1}+s_{2}<0$, so the other factor must be hyperbolic.

With this result in hand, we can now solve the existence and uniqueness problems for Kähler metrics of constant negative scalar curvature on minimal ruled surfaces.

Theorem 4. Let $E \rightarrow \Sigma$ be a rank 2 holomorphic vector bundle over a compact complex curve, and let $(M, J)=\mathbb{P}(E)$ be the total space of the associated $\mathbb{C P}_{1}$-bundle. Let $[\omega]$ be a Kähler class on $M$ with $c_{1} \cdot[\omega]<0$. Then $[\omega]$ contains a Kähler metric of constant scalar curvature iff $E$ is a polystable vector bundle. Moreover, when such a metric exists, it is unique modulo biholomorphisms of $(M, J)$.

Proof. Let us begin by reminding the reader that $\int s d \mu=4 \pi c_{1} \cdot[\omega]$ for any Kähler metric in $[\omega]$, so the $c_{1} \cdot[\omega]<0$ hypothesis exactly limits our discussion to Kähler metrics of constant negative scalar curvature. Note 
that Kähler classes with $c_{1} \cdot[\omega]<0$ will exist on the ruled surface $M \rightarrow \Sigma$ iff $\Sigma$ has genus $\geq 2$.

Now recall that a vector bundle $E$ is said to be polystable (or sometimes quasi-stable) if it is a semi-stable bundle of the form $E=\bigoplus_{j=1}^{n} E_{j}$, where the $E_{j}$ are stable vector bundles. A landmark result of Narasimhan and Seshadri [8] asserts that, for bundles over a Riemann surface, polystability is equivalent to the existence of a flat projective unitary connection on $\mathbb{P}(E)$. If $E$ is polystable, we thus have $(M, J)=\Sigma \times_{\rho} \mathbb{C P}_{1}$ for a representation $\rho: \pi_{1}(\Sigma) \rightarrow P S U(2)=S O(3)$ of the fundamental group which is unique up to conjugation in $S O(3)$. When this happens, local products of constant curvature Käler metrics on $\Sigma$ and $\mathbb{C P}_{1}$ provide us with a constant scalar curvature Kähler metric in each Kähler class. Our task is therefore to show that any Kähler metric on $\mathbb{P}(E)$ with $s=$ const $<0$ is necessarily of this form.

To this end, assume that $g$ is a Kähler metric of constant negative scalar curvature on $(M, J)$, and recall that Theorem 3 tells us that the universal cover of $(M, g)$ must be a product $S^{2} \times \mathcal{H}^{2}$ of spaces of constant curvature. Once the factors are correctly oriented, moreover, the product complex structure will necessarily agree with the lift of $J$ because the holonomy of $S^{2} \times \mathcal{H}^{2}$ is $U(1) \times U(1)$. Now $\pi_{1}(M)=\pi_{1}(\Sigma)$ acts on $S^{2} \times \mathcal{H}^{2}$ by holomorphic isometries, and thus sends any holomorphic curve $S^{2} \times$ $\{\mathrm{pt}\}$ to another curve of this form-after all, these are the only compact complex curves in $\mathbb{C P}_{1} \times \mathcal{H}^{2}$ ! The induced action on $\mathcal{H}^{2}$ is, moreover, free and proper, since $S^{2}$ is compact and every rotation of $S^{2}$ has a fixed point. Thus $\mathbb{P}(E)=\left(S^{2} \times \mathcal{H}^{2}\right) / \pi_{1}(\Sigma)$ is biholomorphic to $\tilde{\Sigma} \times{ }_{\rho} \mathbb{C P}_{1}$ for some compact Riemann surface $\tilde{\Sigma}$ and some representation $\rho: \pi_{1}(\tilde{\Sigma}) \rightarrow$ $P S U(2)=S O(3)$. But since the fibers of $\tilde{\Sigma} \times_{\rho} \mathbb{C P}_{1} \rightarrow \tilde{\Sigma}$ are the only rational curves in $(M, J)$, there is a biholomorphism $\Sigma \rightarrow \tilde{\Sigma}$ such that the diagram

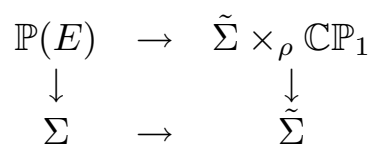

commutes. This gives $E$ a flat unitary projective connection, and so shows that $E$ is polystable.

The same reasoning can easily be applied to other complex surfaces with orientation-reversing diffeomorphisms. For example, on the product $\Sigma_{1} \times \Sigma_{2}$ of two Riemann surfaces of positive genus, any product of constant curvature metrics is the unique constant scalar curvature metric in its Kähler class. Related results have been proved by Leung [7]. 
Corollary 1. Let $E \rightarrow \Sigma$ be a rank 2 holomorphic vector bundle over a compact complex curve, and let $(M, J)=\mathbb{P}(E)$. Let $[\omega]$ be a Kähler class on $M$ with $c_{1} \cdot[\omega]<0$. If $[\omega]$ contains an extremal Kähler metric, then $E$ is either stable, or else is the direct sum $L_{1} \oplus L_{2}$ of a pair of holomorphic line bundles.

Proof. By Theorem 4, we may assume the scalar curvature $s$ of our extremal metric $g$ is nonconstant. Then the isometry group of $g$ is nontrivial, since $J \operatorname{grad}_{g} s$ is a Killing field. Hence there is a Killing field on $M$ which generates a nontrivial $U(1)$-action by biholomorphisms. Since $\Sigma$ has genus $\geq 2$, and so admits no nontrivial holomorphic vector field, this action preserves the fibers of $M \rightarrow \Sigma$, and has 2 distinct fixed points in each fiber. The corresponding linear subspaces of $E$ then give the desired direct sum decomposition.

\section{Acknowledgement}

The author would like to thank Christina Tønnesen for some very useful conversations.

\section{References}

1. D. Burns and P. de Bartolomeis, Stability of Vector Bundles and Extremal Metrics, Inv. Math. 92 (1988), 403-407.

2. E. Calabi, Extremal Kähler Metrics, Seminar on Differential Geometry, ed. S.-T. Yau, Princeton, 1982.

3. R. Friedman and J. Morgan, Algebraic Surfaces and Seiberg-Witten Invariants, preprint.

4. P. Kronheimer and T. Mrowka, The Genus of Embedded Surfaces in the Complex Projective Plane, Math. Res. Lett. 1 (1994), 797-808.

5. C. LeBrun, Einstein Metrics and Mostow Rigidity, Math. Res. Letts. 2 (1995), 1-8.

6. - On the Scalar Curvature of Complex Surfaces, Geom. Func. An. 5 (1995), 619-628.

7. N. C. Leung, Seiberg-Witten Invariants and Uniformization, preprint.

8. M. S. Narasimhan and C. S. Seshadri, Stable and Unitary Vector Bundles on a Compact Riemann Surface, Ann. Math. 85 (1965), 540-567.

9. C. H. Taubes, The Seiberg-Witten invariants and symplectic forms, Math. Res. Letts. 1 (1994), 809-822.

10. E. Witten, Monopoles and Four-Manifolds, Math. Res. Lett. 1 (1994), 769-796.

Department of Mathematics, State University of New York, Stony Brook, NY 11794-3651

E-mail address: claude@math.sunysb.edu 